\title{
Correction to: Method for Generating a Discrete Fracture Network from Microseismic Data and Its Application in Analyzing the Permeability of Rock Masses: A Case Study
}

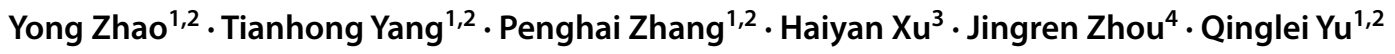

Published online: 8 February 2019

(c) Springer-Verlag GmbH Austria, part of Springer Nature 2019

\section{Correction to: Rock Mechanics and Rock Engineering https://doi.org/10.1007/s00603-018-1712-x}

This paper, not properly checked by the authors when receiving the proofs, is in need of corrections as indicated below.

1. In the second paragraph of Sect. 2.2.3 "Solution case", the second sentence was written as: "The blue and red parts in Fig. 4 indicate the tensile areas, and the white parts indicate the compressive areas.". It should be corrected as: "The blue and red parts in Fig. 4 indicate the compression areas, and the white parts indicate the expansion areas.".

2. In the second paragraph of the title of Fig. 4, the second sentence was written as: "The red, blue and yellow arrows on the beachballs indicate the $P$-axis, $B$-axis and $T$-axis, respectively.". It should be corrected as: "The red, blue and yellow arrows on the beachballs indicate the $T$-axis, $B$-axis and $P$-axis, respectively.".

3. In the third paragraph of Sect. 2.2.3 "Solution case", the first sentence was written as: ", and $\sigma_{1}, \sigma_{2}$ and $\sigma_{3}$ have

The original article can be found online at https://doi.org/10.1007/ s00603-018-1712-x.

Tianhong Yang

yangtianhong@mail.neu.edu.cn

Yong Zhao

zhaoyongrock@163.com

1 Key Laboratory of Ministry of Education on Safe Mining of Deep Metal Mines, Northeastern University, Shenyang 110819, Liaoning, China

2 Center for Rock Instability and Seismicity Research, Northeastern University, Shenyang 110819, Liaoning, China

3 College of Information Science and Engineering, Northeastern University, Shenyang 110819, Liaoning, China

4 College of Water Resources and Hydropower, Sichuan University, Chengdu 610065, Sichuan, China azimuths and plunges of $77.5^{\circ}$ and $90^{\circ}, 65^{\circ}$ and $0^{\circ}$ and $155^{\circ}$ and $0^{\circ}$, respectively.". It should be corrected as: "and $\sigma_{3}, \sigma_{1}$, and $\sigma_{2}$ have azimuths and plunges of $77.5^{\circ}$ and $90^{\circ}, 65^{\circ}$ and $0^{\circ}$ and $155^{\circ}$ and $0^{\circ}$, respectively.".

Publisher's Note Springer Nature remains neutral with regard to jurisdictional claims in published maps and institutional affiliations. 